FORUM EARTH SCIENCE

The timing of climate change

An innovative assessment of climate change calculates the year in which ongoing warming will surpass the limits of historical climate variability. Three experts explain this calculation's significance compared with conventional approaches, and its relevance to Earth's biodiversity. SEE ARTICLE P.183

\section{THE PAPER IN BRIEF}

- In this issue, Mora et al. ${ }^{1}$ present a novel characterization of climate change.

- The assessment is based on an estimate of when ongoing warming will continuously exceed the bounds of historical climate variability.
- Depending on the volumes of greenhouse gases emitted in the future, this could, averaged globally, occur as early as midcentury.

- The change will occur first in the tropics, for which historical variability is low and biodiversity is highest (Fig. 1).

\section{Beyond historical variability}

\section{CHRIS HUNTINGFORD \& LINA MERCADO}

$\mathrm{T}_{\mathrm{e}}^{\mathrm{h}}$ he debate on climate change focuses almost exclusively on mean meteorological adjustments likely to occur by the years 2020, 2050 and 2100. The first of these is an immediate timescale, which society may consider preparing for now. The year 2050 is a time by which significant change might occur, and large-scale planning to counteract this should, arguably, commence soon. It may take the next three decades to implement major adaptation schemes, or to devise mitigation solutions that could potentially include geoengineering.

Mora and colleagues' study provides two refreshing advances to our understanding of climate change. First, it concentrates on climate variability instead of overall mean change. Second, it identifies the year in which climate change will cross the threshold whereby events that are currently regarded as extreme become the norm. This may seem trivial - the authors are simply reinterpreting and inverting the same 'change versus year diagrams', but for a prescribed threshold. However, the subtle change of using scientifically based potential years of concern, as opposed to standard prescribed years such as 2050, may refocus the climate-policy debate.

Increasing emphasis is being placed on climate models in the prediction of not only average changes in weather conditions, but also properties of extreme climatic events. Risks associated with any increased frequency of such events have been well documented in both academic circles and the mainstream media. For instance, the hydrological cycle could adjust such that some regions receive more pronounced rainfall events, triggering flooding and landslides; measurements already suggest rainfall intensification in some areas ${ }^{2}$. Elsewhere, more frequent droughts could reduce crop yields. And how should cities be designed to deal with recurrent heatwaves? Such issues led the Intergovernmental Panel on Climate Change to commission a specific report on the current understanding of climate extremes ${ }^{3}$.

There are also concerns about how these extremes might influence ecosystems and biodiversity. Mora and colleagues' analysis adopts an adaptation hypothesis that the bounds of ecosystem resilience might be tightly tied to historical exposure to climatic variation. If this proves to be the case, it could have surprising implications. For regions of relatively low interannual variability, such as the tropics, ecological damage could occur in response to a smaller amount of climate change than previously thought. This might be true even in the absence of an increase in extremes, because average change alone could cause persistent crossing of historical warming limits. Indeed, recent work ${ }^{4}$ suggests that, in the past few decades, interannual temperature variation has actually decreased for the tropics. In other places, more frequent extremes may soon routinely push beyond the limits of adaptation. Some argue that more extremes are already starting to decrease the ability of terrestrial ecosystems to sequester carbon and offset carbon dioxide emissions ${ }^{5}$.

For a 'business-as-usual' emissions scenario, averaging across all regions and climate models, Mora et al. estimate 2047 to be the mean year in which warming will begin to reach almost continuously beyond historical bounds, potentially affecting ecosystem health drastically. If the bounds on adaptation are defined by pre-industrial variability only, an even earlier date applies. Although continuing improvements to climate models will refine these timings, there is a more fundamental problem. At present, very few largescale ecological models account for potential acclimation to future climatic conditions. This is a short-term adjustment - occurring over minutes to weeks - made by an organism in response to any new climatic conditions to improve its performance, thereby offsetting possible damage. The main reason for this modelling omission is a general paucity of physiological data, especially for tropical ecosystems, to parameterize such effects should they exist. Arguably, even less seems to be known about large-scale climate-biodiversity interactions and feedbacks, preventing interpretation of climate-model diagnostics in the context of biodiversity stability.

Chris Huntingford is at the Centre for Ecology \& Hydrology, Wallingford OX10 8BB, UK. Lina Mercado is at the College of Life and Environmental Sciences, University of Exeter, Exeter EX4 4RJ, UK.

e-mails:chg@ceh.ac.uk;

l.mercado@exeter.ac.uk

\section{Tropical diversity countdown}

\section{ERIC POST}

$\mathbf{R}$ ather than emphasizing general projecRtions of expected temperature increases over the next century, Mora et al. focus on estimating precisely when continuous deviation from mean climatic conditions that have prevailed over the past century and a half is likely to occur. Putting numbers on their 'timing of climate departure' may prove a tricky business because detractors will surely concentrate on the precise estimates of years of 


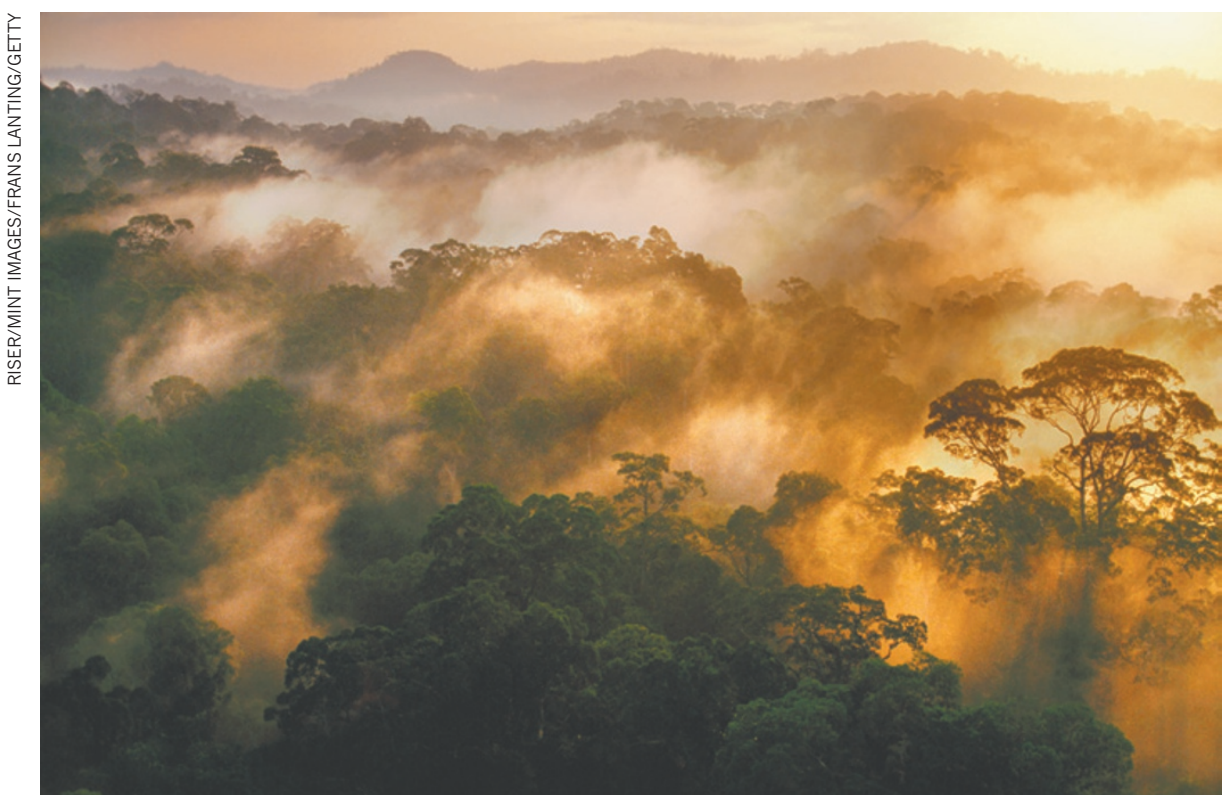

Figure 1 | The tropical rainforest. Tropical regions, which are characterized by low levels of climatic variability, may be the first to experience deviation beyond the bounds of long-term past climatic conditions ${ }^{1}$

departure so derived. But the approach provides an important complement to the estimation of climate-change velocities ${ }^{6}$, and, when applied regionally, may have implications for understanding the role of climate change in biodiversity loss.

As all ecologists know, the increase in species diversity from the poles to the tropics is matched by increases in temperature and precipitation, and by a decline in seasonal variation in climate ${ }^{7}$. However, the importance of climate history and climatic gradients in the generation of diversity gradients remains obscure $^{8}$, despite improvements in model complexity ${ }^{9}$.

Because the processes that generate and maintain diversity operate over disparate scales of space and time, we might expect the roles of variation in climatic conditions through space and time to differ between those processes as well ${ }^{10}$. Hence, where the maintenance of greater diversity in the tropics is concerned, the comparative stability of climatic conditions in the tropics relative to temperate and polar regions may be invoked more convincingly. This is because tropical species, especially the highly diverse group known as terrestrial ectotherms - which includes amphibians and reptiles - are characterized by narrow ranges of thermal tolerance. This indicates that these creatures have a high degree of specialization for relatively constant thermal conditions compared with organisms adapted to environmental conditions at higher latitudes ${ }^{11}$.

Mora and colleagues' study suggests that it is these low-latitude specialists that will be the first to experience deviation of climatic conditions from long-term average conditions. Therefore, regardless of the precision of the estimated timing of climate departure itself, the tropics will reach this state first, an intriguing insight. This conclusion derives from the authors' emphasis on deviation from existing conditions as opposed to the absolute magnitude of expected warming, which is and will continue to be greatest at high northern latitudes.

Departure is expected to occur first in the tropics precisely because this region has had

\section{PALAEONTOLOGY}

\section{MATT FRIEDMAN \& MARTIN D. BRAZEAU}

$\int$ awed vertebrates, or gnathostomes, comprise the vast majority of backboned animals. Understanding their great success and the evolution of their anatomical marvels lies in reconstructing the nature of their most recent common ancestor. An entrenched assumption of classical comparative anatomy ${ }^{1}$, which has gained some recent palaeontological support ${ }^{2,3}$, is that this precursor resembled a shark. This hypothesis implies that the anatomical peculiarities of bony fishes - the group that would eventually give rise to ourselves - are specializations that arose later in a relatively invariable climate over the period 1860-2005, which the authors use as a baseline. Thus, even with relatively little absolute warming, conditions in the tropics will rapidly deviate from historical baseline variability. And it is this deviation that makes sense biologically when considering the conservation implications of climate change for species with narrow physiological bounds of thermal tolerance $^{11}$. If the assessment by Mora et al. proves accurate, conservation practitioners take heed - the climate-change race is not only on, it is fixed, with the extinction finish line looming closest for the tropics.

Eric Post is in the Polar Center and Department of Biology, Pennsylvania State University, University Park, Pennsylvania 16802, USA.

e-mail:esp10@psu.edu

1. Mora, C et al. Nature 502, 183-187 (2013).

2. Min, S.-K., Zhang, X., Zwiers, F. W. \& Hegerl, G. C. Nature 470, 378-381 (2011).

3. IPCC Managing the Risks of Extreme Events and Disasters to Advance Climate Change Adaptation (eds Field, C. B. et al.) (Cambridge Univ. Press, 2012)

4. Huntingford, C., Jones, P. D., Livina, V. N., Lenton, T. M. \& Cox, P. M. Nature 500, 327-330 (2013).

5. Reichstein, M. et al. Nature 500, 287-295 (2013)

6. Loarie, S. R. et al. Nature 462, 1052-1055 (2009)

7. Gaston, K. J. Nature 405, 220-227 (2000).

8. Pimm, S. L. \& Brown, J. H. Science $\mathbf{3 0 4 , 8 3 1 - 8 3 3}$ (2004).

9. Gotelli, N. J. et al. Ecol. Lett. 12, 873-886 (2009)

10.Post, E. Ecology of Climate Change: The Importance of Biotic Interactions (Princeton Univ. Press, 2013).

11. Tewksbury, J. J., Huey, R. B. \& Deutsch, C. A. Science 320, 1296-1297 (2008).

\section{A jaw-dropping fossil fish}

The ancestors of modern jawed vertebrates are commonly portrayed as fishes with a shark-like appearance. But a stunning fossil discovery from China puts a new face on the original jawed vertebrate. SEE ARTICLE P.188

vertebrate evolutionary history. On page 188 of this issue, Zhu et al. ${ }^{4}$ present a 419-millionyear-old fossil and accompanying analysis that turn this view on its head*.

The deepest split in the family tree of modern gnathostomes divides the cartilaginous fishes (chondrichthyans, which include sharks, rays and ratfishes) from the much more speciesrich bony fishes (osteichthyans, comprising ray-finned fishes, lungfishes, coelacanths and terrestrial vertebrates). Among other differences, the two groups show contrasting conditions of the external skeleton: cartilaginous

*This article and the paper under discussion were published online on 25 September 2013. 